\title{
Thermal Effects on the Characteristics of AlGaAs/ GaAs Heterojunction Bipolar Transistors Using Two-Dimensional Numerical Simulation
}

\author{
Lee L. Liou, Member, IEEE, John L. Ebel, and Chern I. Huang, Member, IEEE
}

\begin{abstract}
Simulations incorporating the spatial distributions of the energy band and temperature have been used to study AlGaAs/GaAs heterojunction bipolar transistor characteristics. The coupled heat transfer and conventional device carrier transport equations were solved numerically in two dimensions using both the voltage and current boundary conditions applied on the base contact. An equivalent thermal resistance model was used to establish the temperature boundary condition between the electrically active device and the relatively large thermally active semi-insulating substrate. The negative differential resistance and the reduction of the base-emitter voltage for a constant base current in the active region are caused by the thermal effects. The differential current gain and cutoff frequency are decreased when the transistor is operated at high power levels. The temperature distribution of the transistor operated in the active region shows a maximum temperature occurring at the collector region right beneath the emitter mesa. When the transistor operated in the saturation region, the emitter contact region may be at a slightly lower temperature than the heat sink temperature. This thermoelectric cooling effect reults from the utilization of the thermodynamically compatible current and energy flow formulations in which the energy band discontinuities are part of the thermoelectric power.
\end{abstract}

\section{NOMENCLATURE}

C Net doping density.

$d$ Thickness of substrate.

$E_{c} \quad$ Conduction band edge.

$E_{v} \quad$ Valence band edge.

$E_{g} \quad$ Energy bandgap.

$E_{f n} \quad$ Quasi-Fermi energy for electrons.

$E_{f p} \quad$ Quasi-Fermi energy for holes.

$F_{j} \quad$ Fermi-Dirac integral of the $j$ th order given by

$$
F_{j}(y)=\frac{1}{\Gamma(j+1)} \int_{0}^{\infty} \frac{x^{j} d x}{1+\exp (x-y)} .
$$

$I_{b} \quad$ Base current density $(\mathrm{A} / \mathrm{m})$.

$I_{c} \quad$ Collector current density $(\mathrm{A} / \mathrm{m})$.

$J_{E} \quad$ Energy flow $\left(\mathrm{W} / \mathrm{m}^{2}\right)$.

Manuscript received April 23, 1992; revised August 12, 1992. This work was supported in part by the Air Force Office of Scientific Research. The review of this paper was arranged by Associate Editor A. H. Marshak.

The authors are with the Solid State Electronics Directorate, Wright Laboratory, Wright-Patterson Air Force Base, OH 45433-6543.

IEEE Log Number 9204304
$J_{n} \quad$ Electron current density.

$J_{p} \quad$ Hole current density.

$k \quad$ Boltzmann's constant.

$l_{x} \quad x$ dimension of device.

$l_{y} \quad y$ dimension of device.

$L_{x} \quad x$ dimension of substrate.

$L_{y} \quad y$ dimension of substrate.

$n \quad$ Electron density.

$N_{c} \quad$ Equivalent density of states for conduction band.

$N_{v} \quad$ Equivalent density of states for valence band.

$p \quad$ Hole density.

$P \quad$ Power dissipation per unit volume $\left(\mathrm{W} / \mathrm{m}^{3}\right)$.

$q \quad$ Elementary charge.

$Q \quad$ Assumed uniform energy flow of device to the substrate $\left(\mathrm{W} / \mathrm{m}^{2}\right)$.

$R \quad$ Net recombination rate.

$R_{\text {th }} \quad$ Specific thermal resistance between the bottom of the device and the heat sink $\left(\mathrm{K} \cdot \mathrm{m}^{2} / \mathrm{W}\right)$.

$T$ Temperature (K).

$T_{0} \quad$ Heat sink temperature, $300 \mathrm{~K}$

$V_{b e} \quad$ Applied base-emitter voltage.

$V_{c e} \quad$ Applied collector-emitter voltage.

$\alpha(y)=3 F_{2}(y) / F_{1}(y)$.

$\alpha_{n} \quad=\alpha\left(\eta_{n}\right)$.

$\alpha_{p}=\alpha\left(\eta_{p}\right)$.

$\beta(y)=F_{0.5}(y) / F_{-0.5}(y)$.

$\beta_{n}=\beta\left(\eta_{n}\right)$.

$\beta_{p}=\beta\left(\eta_{p}\right)$.

$\Delta E_{c}$ Conduction band discontinuity at heterojunction.

$\Delta E_{c}^{*}$ Effective conduction band discontinuity at heterojunction.

Dielectric constant.

$\theta$ Temperature calculated using a temperature-independent thermal conductivity.

$\eta_{n} \quad=\left(E_{f n}-E_{c}\right) /(k T)$.

$\eta_{p}=\left(E_{v}-E_{f p}\right) /(k T)$.

$\kappa \quad$ Thermal conductivity $(\mathrm{W} /(\mathrm{m} \cdot \mathrm{K}))$.

$\kappa_{0} \quad$ Thermal conductivity at heat sink temperature $(\mathrm{W} /(\mathrm{m} \cdot \mathrm{K}))$.

$\mu_{n} \quad$ Electron mobility.

$\mu_{p} \quad$ Hole mobility.

$\chi \quad$ Electron affinity.

$\psi \quad$ Electrostatic potential. 


\section{INTRODUCTION}

$\mathrm{H}$ ETEROJUNCTION bipolar transistors (HBT's) are being developed for microwave high-power applications. Power dissipation under high-current operation strongly affects transistor characteristics and must be considered in the device design. Thermal effects on HBT's have been studied both theoretically and experimentally. Several techniques have been used to measure the device junction temperature. One method relies on the dependence of base-emitter voltage on temperature [1]-[3]. Others use the dependence of common emitter current gain with temperature [4], [5]. The measured junction temperature in these studies can only be regarded as a macroscopic average quantity. Gao et al. [6] studied the thermal design considerations of high-power $\mathrm{AlGaAs} / \mathrm{GaAs}$ HBT's. In their study, the HBT's were treated as heat sources on the top of a substrate, and the power density (energy flow) distribution function was calculated. The temperature distribution on the top surface of the substrate was then calculated by solving the heat transfer equation. The temperature distribution and thermal related properties such as device power handling capability and thermal instability as functions of the substrate geometry and layout design of the HBT's with multiple emitter fingers could be deduced. However, the detailed temperature distribution in the device was not considered.

Detailed temperature distribution in an HBT can be calculated numerically by solving the coupled conventional device carrier transport (Poisson's and continuity equations for electrons and holes) and heat transfer equations. Such a study has been performed on power GaAs metalsemiconductor field-effect transistors (MESFET's) [7]. The current-voltage $(I-V)$ characteristics have shown negative differential resistance (NDR) in the saturation region. Using a one-dimensional (1D) numerical simulation, the $I-V$ characteristics of an HBT also showed NDR [8]. However, this 1D work, assuming the collector contact being at the heat-sink temperature, did not consider the heat dissipation through the substrate. Proper treatment of such a more realistic situation is important, since the substrate is usually constituting a significant part of the total thermal resistance. The present work, an extension of the previous 1D simulation study, studies the thermal effects on the device $I-V$ characteristics using a $2 \mathrm{D}$ numerical simulation taking into account the thermal resistance of the substrate. The resulting temperature distributions in the device show the normally considered temperature rise due to Joule heating as well as a possible thermoelectric cooling effect due to the heterojunction at the emitter cap and the emitter barrier.

\section{Calculation}

In the present study, the device geometry and doping concentration are shown in Fig. 1(a). We further assume a simple situation, as shown in Fig. 1(b), in which the HBT is sitting at the center of a relatively large substrate. The temperature distribution in an HBT is strongly af-

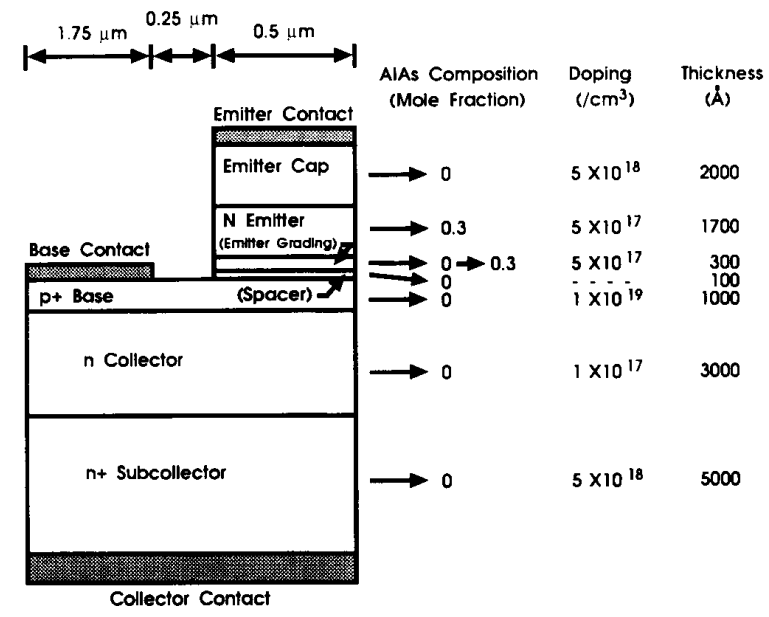

(a)
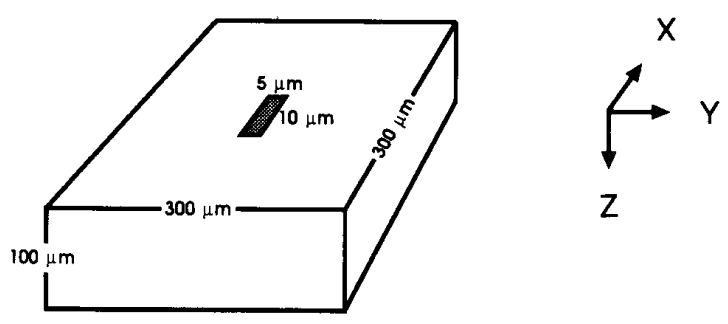

(b)

Fig. 1. (a) Schematic cross section of half of the HBT device. Due to the symmetry, only half of the device is simulated. (b) Position of the device on GaAs substrate plate.

fected by not only the operating conditions of the device but also the thermal properties and geometry of the substrate. The dimension of the thermally active region is usually much larger than the electrically active device. It would not be efficient to solve the coupled electric (driftdiffusion carrier transport) and heat transfer equations for the whole system. Following the strategy of Ghione and Naldi [7] in their work on the power GaAs MESFET's, we solve the coupled equations for the intrinsic HBT selfconsistently including a temperature boundary condition at the interface between the device and the substrate. This temperature boundary condition is modeled using an equivalent thermal resistance concept and will be elaborated further later.

In steady state, the equations governing the intrinsic device are given by

$$
\begin{aligned}
\nabla \cdot(\epsilon \nabla \psi) & =q(n-p-C) \\
\nabla \cdot \vec{J}_{n} & =q R \\
\nabla \cdot \vec{J}_{p} & =-q R \\
\nabla \cdot[\kappa(T) \nabla T] & =-P=\nabla \cdot \vec{J}_{E}
\end{aligned}
$$

where the symbols and those used hereafter are specified in the Nomenclature Section. 
The general drift-diffusion model including the nonuniform spatial distributions of the energy band, degeneracy factor, and temperature will be applied in the present study. The semiconductor current-flow equation including a temperature gradient term has been derived by Stratton [9]. The coefficient of this temperature gradient term depends on the free carrier relaxation time $\tau$ which in turn is a function of the carrier energy above the band edge. Marshak [10] has derived a generalized drift and diffusion current equation for heterostructure semiconductor. His calculation has resulted in two extra terms due to heterostructure in the electron and hole current equations besides the conventional drift and diffusion terms. One term is proportional to the gradient of the electron affinity for the electron current, and the gradient of the sum of electron affinity and bandgap for the hole current. The other is proportional to the gradient of the equivalent density of states for the respective energy bands for the electron and hole currents. The electron and hole current densities $J_{n}$ and $J_{p}$ are given by

$$
\begin{aligned}
\vec{J}_{n}= & \mu_{n} \beta_{n} k T \nabla n-q n \mu_{n}\left[\nabla\left(\psi+\frac{\chi}{q}\right)\right. \\
& \left.+\beta_{n} \frac{k T}{q} \frac{\nabla N_{c}}{N_{c}}-\alpha_{n} \nabla\left(\frac{k T}{q}\right)\right] \\
\vec{J}_{p}= & -\mu_{p} \beta_{p} k T \nabla p-q p \mu_{p}\left[\nabla\left(\psi+\frac{\chi+E_{g}}{q}\right)\right. \\
& \left.-\beta_{p} \frac{k T}{q} \frac{\nabla N_{v}}{N_{v}}+\alpha_{p} \nabla\left(\frac{k T}{q}\right)\right] .
\end{aligned}
$$

The first term on the right hand side (RHS) of (5) and (6) is the diffusion component where the diffusion coefficient including the degeneracy factor $\beta_{n, p}$ is $\mu \beta_{n, p} k T / q$. The second term is the generalized drift component which is a summation of the contributions from the electric field, band structure, and temperature gradient. Assuming the free carrier relaxation time is proportional to the square root of the energy, the coefficients $\alpha_{n, p}=$ $3 F_{2}\left(\eta_{n, p}\right) / F_{1}\left(\eta_{n, p}\right)$ in (5) and in (6) are obtained. The main temperature gradient in the AlGaAs/GaAs HBT device usually resides in the GaAs collector region, where the doping density is less than $2 \times 10^{17} / \mathrm{cm}^{3}$, and the polaroptical mode is the dominant scattering mechanism. A dependency of the relaxation time on the square root power of carrier energy is a reasonable approximation in this GaAs collector region [12]. For the region where acoustic phonon scattering is the dominant scattering mechanism, the relaxation time is inversely proportional to the square root of the carrier energy, and $\alpha_{n, p}=2 F_{1}\left(\eta_{n, p}\right) / F_{0}\left(\eta_{n, p}\right)$, which were used in [11]. For the region where the dominant scattering mechanism is the ionized impurity scattering, the relaxation time is proportional to the three halves power of the carrier energy, and $\alpha_{n, p}=$ $4 F_{3}\left(\eta_{n, p}\right) / F_{2}\left(\eta_{n, p}\right)$.
One of the basic assumptions made in the drift-diffusion model is that the electrons and holes are in thermal equilibrium locally with the host lattice (heat loss due to radiation and convection with the ambient environment is not considered here). This assumption makes the semiconductor, composed of the subsystems of free carriers and lattice, a thermodynamic system. Temperature, besides the quasi-Fermi levels for electrons and holes, is one of the dynamic variables. Using a rigorous thermodynamics treatment and without an explicit form for the heterostructure, Wachutka [13] is able to show similar electron and hole current equations as (5) and (6), where $k \alpha_{n} / q$ and $k \alpha_{p} / q$ are the heterostructure-excluded thermoelectric power associated with the electrons and holes. Modified slightly, his derivation for the heterostructure leads to the energy flow equation

$$
\begin{aligned}
\vec{J}_{E}= & \left\{-\left[\alpha_{n} k T+E_{c}\right] \frac{\vec{J}_{n}}{q}-\kappa_{n} \nabla T\right\} \\
& +\left\{\left[\alpha_{p} k T-E_{v}\right] \frac{\vec{J}_{p}}{q}-\kappa_{p} \nabla T\right\}
\end{aligned}
$$

where $\vec{J}_{E}$ is the energy flow, $\kappa_{n}$ and $\kappa_{p}$ are the thermal conductivities associated with electron gas and hole gas, respectively. The first term in the two braces on the RHS of (7) is the heuristic energy flow contribution of the electron and hole currents. The remaining terms on the RHS take into account the heat flow associated with the temperature gradient. These temperature gradient terms after the operation of divergence, can be combined with $\kappa$, the lattice thermal conductivity in (4). As discussed by the same author [13], $\kappa_{n}$ and $\kappa_{p}$ are less than $1 \%$ of $\kappa$ for the carrier concentration of less than $10^{19} / \mathrm{cm}^{3}$, and therefore, their contribution to the energy flow is neglected in the present calculation.

The adiabatic temperature condition is applied on the device boundary except at the bottom of the device where the temperature boundary condition is given by

$$
-\kappa(T) \nabla T \cdot \hat{n}=\frac{\left(T-T_{0}\right)}{R_{\mathrm{th}}}
$$

where $\hat{n}$ is the outwardly normal unit vector, $T_{0}$ is the heat sink temperature, and $R_{\mathrm{th}}$ is the equivalent specific thermal resistance of the substrate. The left hand side (LHS) of (8) is the energy flow from the device into the substrate, while the RHS implements the equivalent thermal resistance concept. $R_{\mathrm{th}}$ can be calculated by taking into account the three-dimensional heat flow in the substrate. The calculation of $R_{\mathrm{th}}$ for the situation in Fig. 1(b) is given in the Appendix.

It is important to implement accurate temperature dependence of the material parameters especially for thermal simulation. In this study, the hole mobility $\mu_{p}$ and electron mobilities $\mu_{n}$ in GaAs as functions of temperature, electric field, and doping density are given by [14], 
[15]

$$
\begin{aligned}
& \mu_{p}\left(T, N_{D}+N_{A},|E|\right)=\frac{380(300 / T)^{2.7}}{\left[1+3.17 \times 10^{-17}\left(N_{D}+N_{A}\right)\right]^{0.266}\left(1+|E| / 1.95 \times 10^{4}\right)} \mathrm{cm}^{2} / \mathrm{V} \cdot \mathrm{s} \\
& \mu_{n}\left(T, N_{D}+N_{A},|E|\right)=\frac{\mu_{L}\left(T, N_{D}+N_{A}\right)+v_{\mathrm{sat}}|E|^{3} / E_{C}^{4}}{1+\left(|E| / E_{C}\right)^{4}} \mathrm{~cm}^{2} / \mathrm{V} \cdot \mathrm{s}
\end{aligned}
$$

where $|E|$ is the magnitude of the electric field; $N_{A}$ and $N_{D}$ are the acceptor and donor doping densities; the lowfield mobility is

$$
\begin{aligned}
\mu_{L}(T, & \left.N_{D}+N_{A}\right) \\
\quad= & \frac{7200(300 / T)^{2.3}}{\left[1+5.51 \times 10^{-7}\left(N_{D}+N_{A}\right)\right]^{0.233}} \mathrm{~cm}^{2} / \mathrm{V} \cdot \mathrm{s}
\end{aligned}
$$

the saturation velocity is

$$
v_{\text {sat }}=(1.28-0.0015 T) \times 10^{7} \mathrm{~cm} / \mathrm{s}
$$

and the critical field is

$$
E_{C}=(5.4-T / 215) \mathrm{kV} / \mathrm{cm} \text {. }
$$

$\mu_{n}$ in $\mathrm{Al}_{x} \mathrm{Ga}_{1-x} \mathrm{As}$ is calculated by taking the weighted average of the mobilities in the $\Gamma$ - and $X$-valleys. The weighting factors are the respective carrier populations in both valleys [15]. $\mu_{p}$ in $\mathrm{Al}_{x} \mathrm{Ga}_{1-x}$ As is calculated by assuming the validity of the relaxation time and linear response approximation, in which $\mu_{p}\left(\mathrm{Al}_{x} \mathrm{Ga}_{1-x} \mathrm{As}\right)=$ $\mu_{p}(\mathrm{GaAs}) \cdot\left[m_{p, \mathrm{GaAs}}^{*} / m_{p, \mathrm{AlGAAs}}^{*}\right] \cdot\left[\tau_{p, \text { AIGaAs }} / \tau_{p, \mathrm{GaAs}}\right]$, where $m_{p}^{*}$ and $\tau_{p}$ are the hole effective mass and momentum relaxation time for holes, respectively. The detailed modeling of the electron and hole mobilities in $\mathrm{AlGaAs}$ can be found in [15]. The percentage distributions of the bandgap difference in conduction and valence-band discontinuities between AlGaAs and GaAs were assumed to be $65 \%$ and $35 \%$ for the temperature range of interest in the present calculation. The other material parameters, such as the effective mass and equivalent density of states in the conduction and valence bands and the dielectric constants, were adopted from [16]. The thermal conductivities of GaAs and AlGaAs were taken from [17] and [18], respectively. The Shockley-Reed-Hall recombination with a carrier lifetime of $1 \times 10^{-9} \mathrm{~s}$, assumed temperature-independent, for both electrons and holes was implemented, while the surface recombination was not included.

A central finite difference discretization with nonuniform interval was used. A fully coupled Newton-Raphson numerical method was applied to solve the electrical equations (1)-(3). To begin the simulation, a uniform temperature distribution was assumed. The energy flow was calculated from the solution of electrical equations, and was used in the heat transfer equation (4) for solving the temperature distribution. With the updated temperature distribution, (1)-(3) were solved again. These procedures were repeated until the difference between two successive temperature distributions at every discretized location was smaller than the preset values of $0.03 \mathrm{~K}$ for low current level and $0.3 \mathrm{~K}$ for high current level. To simulate a successive bias point on the same $I-V$ curve, the solution of the previous bias point was used as the initial guess value. The structure in the calculation was discretized into a mesh of $40 \times 120\left(N_{y} \times N_{z}\right)$ points. The required computer storage space is about $45 \mathrm{Mb}$. The simulation was run on a Convex C220 machine, and CPU time for one bias point in $I-V$ characteristics ranges from 10 to 40 min depending on the current levels. Usually higher current level requires a larger number of the iterations, hence a longer computing time.

\section{Results AND Discussion}

The simulations were carried out on an AlGaAs/GaAs HBT using both the voltage and current boundary conditions applied on the base contacts [19]. The calculations were done on the symmetric half of this emitter-mesa HBT shown in Fig. 1(a). The heat-sink temperature was $300 \mathrm{~K}$ in the following calculations.

Using the voltage boundary condition at the base contact, the Gummel plot, i.e., the collector and base current densities $I_{c}$ and $I_{b}$, as functions of base-emitter voltage $V_{b e}$ for a fixed collector-emitter voltage $V_{c e}$ is shown in Fig. 2. $V_{c e}$ is fixed at $3 \mathrm{~V}$ in the isothermal simulation and is ranging from 1 to $5 \mathrm{~V}$ in the thermal simulation. In the region of $I_{c}$ of less than $10^{2} \mathrm{~A} / \mathrm{m}$, both thermal and isothermal results coincide due to insignificant temperature rise in that current range. As $I_{c}$ becomes larger than $10^{2}$ $\mathrm{A} / \mathrm{m}$, both $I_{c}$ and $I_{b}$ deviate from the isothermal results. For large $V_{c e}$, as $V_{b e}$ increases, the ideality factors for both $I_{c}$ and $I_{b}$ can be much less than one before both currents reach saturation levels in which current stays almost constant. In this region, $I_{c}$ is mainly affected by the series resistance in the collector. Increase in $V_{c e}$ causes temperature rise in the collector region. As a result, collector resistance increases and saturation level for $I_{c}$ is slightly smaller when $V_{c e}$ is larger.

The dependence of differential current gain and cutoff frequency on the collector current density with $V_{c e}$ as the variable parameter are shown in Fig. 3(a) and (b). All the curves of the current gain show a maximum. The decrease in the isothermal current gain as $I_{c}$ increases beyond $10^{3}$ A $/ \mathrm{m}$ is due to the Kirk effect or the base pushout effect. As $V_{c e}$ increases, the maximum differential current gain decreases and occurs at a lower $I_{c}$. Two reasons may be used to explain this trend. One is that the threshold of the Kirk effect is proportional to the collector doping density as well as the electron saturation velocity in the collector space-charge region. The threshold level decreases as $V_{c e}$ increases, since the saturation velocity becomes reduced 


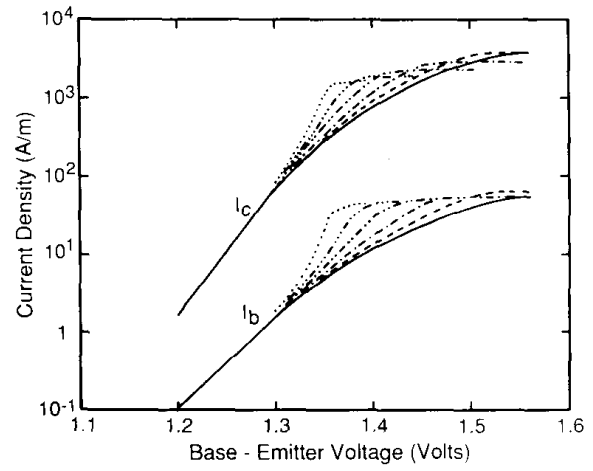

Fig. 2. The calculated Gummel plot. (- $)$ is the isothermal thermal simulation results with $V_{c e}=3 \mathrm{~V} .(---),(-\cdot-),(-\cdots-),(-\cdots-)$, and $(\cdots)$ are the thermal simulation results with $V_{u e}=1,2,3,4$, and 5 $\mathrm{V}$, respectively.

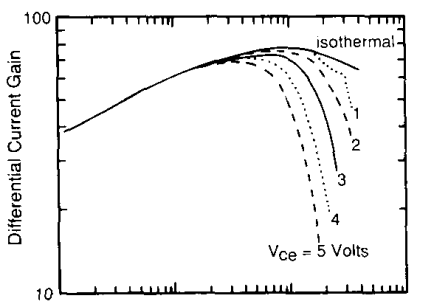

(a)

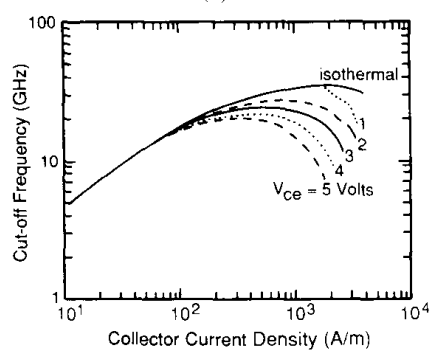

(b)

Fig. 3. (a) The differential current gain as a function of collector current density with $V_{c e}$ as the variable parameter. (b) The cutoff frequency as a function of collector current density with $V_{c e}$ as the variable parameter. The isothermal results are also shown.

at higher temperature. The other factor causing the significant drop of the differential current gain in the highcurrent region is the reduction of the emitter injection efficiency when the base-emitter junction temperature is high. The hole occupation probability would spread out in a wider energy range in the valence band at a higher temperature. As a result, the base current, mainly the thermionic emission-diffusion current in the emitter barrier, increases and the current gain drops [20], [21]. A similar trend in the cutoff frequency dependency on $I_{c}$ and $V_{c e}$ can also be explained by the Kirk effect with which the equivalent base width increases causing an increase in the base transit time. When $V_{c e}$ increases, the carrier transit time through the equivalent base and base-collector space-charge region becomes larger due to a lower electron mobility at higher temperatures.

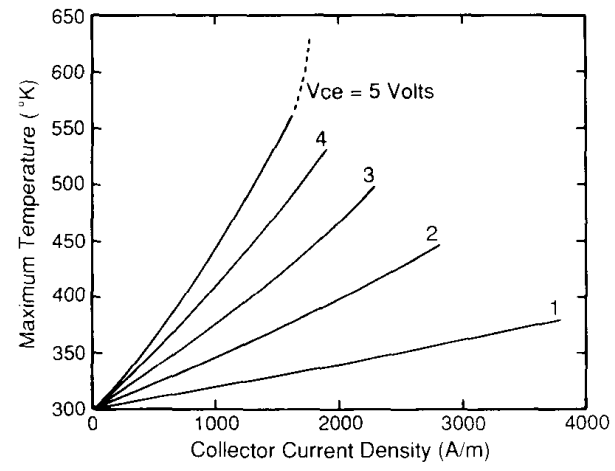

Fig. 4. The maximum temperature in the device as a function of collector current density with $V_{c c}$ as the variable parameter.

The dependence of the maximum temperature in the device on the collector current density with $V_{c e}$ at the variable parameter is illustrated in Fig. 4. As collector current increases, so do the power dissipation and device temperature. With respect to the maximum temperature in the device, if the rate of power dissipation increase exceeds that of the heat flow through the thermal resistance, the thermal instability (also known as thermal runaway, defined by $d T_{\max } / d I_{c} \rightarrow \infty$ ) occurs. The temperature may increase without limit until the device is destroyed. With the present numerical scheme, the simulation with a higher temperature rise requires a longer computation time. Convergent solutions were not obtained for the device with a maximum temperature larger than $580 \mathrm{~K}$. By extrapolating the curve for $V_{c e}=5 \mathrm{~V}$ in Fig. 4 , the thermal runaway in the present case may occur around a collector current density of $2000 \mathrm{~A} / \mathrm{m}$ (corresponding to $2 \times 10^{5} \mathrm{~A} / \mathrm{cm}^{2}$ of the current density for an emitter width of $1 \mu \mathrm{m}$ ). Thermal runaway for the device operated with a smaller $V_{c e}$ occurs at a higher collector current.

Using the current boundary condition, the HBT common-emitter $I-V$ characteristics are shown in Fig. 5(a). While the normal base modulation is observed utilizing the isothermal simulation, the NDR in the active region (i.e., $V_{c e}>0.5 \mathrm{~V}$ ) is produced by the thermal model. The NDR occurs mainly due to the reduction of carrier mobility as the temperature increases when the device is under high current operation. The corresponding base-emitter voltage $V_{b e}$ as a function of collector-emitter $V_{c e}$ with the base current $I_{b}$ as the variable parameter is shown in Fig. 5(b). The isothermal simulation results are also shown for comparison. For a constant base current, the thermal effects cause $V_{b e}$ to decrease as $V_{c e}$ increases in the active region. This inverse dependency of $V_{b e}$ on $V_{c e}$ has also been seen previously in Fig. 2. An HBT with the same structural parameters as shown in Fig. 1 has been fabricated. The thermal resistance of the device was measured indirectly to be $2.9 \mathrm{~K} / \mathrm{mW}$ [4]. Estimated from the simulation results of $I_{b}=20 \mathrm{~A} / \mathrm{m}$ and $V_{c e}=3 \mathrm{~V}$, which gives a power of $38 \mathrm{~mW}$, and the calculated maximum temperature rise of $100 \mathrm{~K}$ (see Fig. 6(a) in the following), the thermal resistance is about $2.6 \mathrm{~K} / \mathrm{mW}$. These thermal 


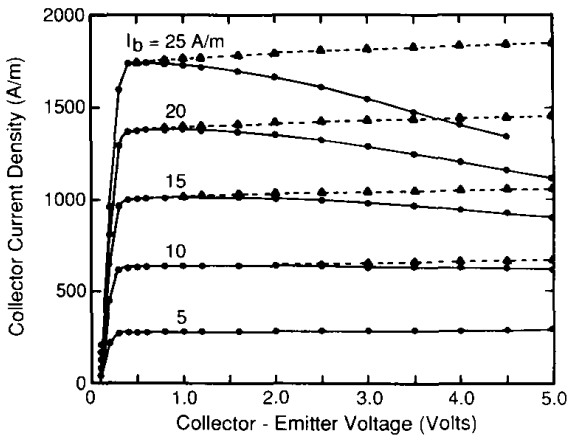

(a)

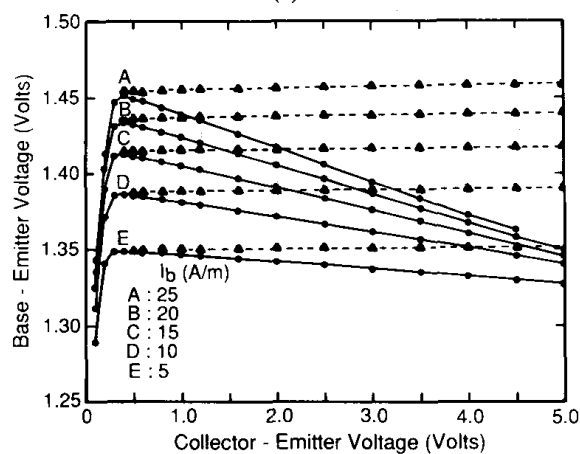

(b)

Fig. 5. (a) The calculated collector current density as a function of collector-emitter voltage with the base current density $I_{b}=5,10,15,20$, and $25 \mathrm{~A} / \mathrm{m}$. The lines with $(\bullet)$ and the lines with $(\Delta)$ are the results with and without thermal model incorporated, respectively. (b) The calculated base-emitter voltage as a function of collector-emitter voltage with the base current density $I_{b}=5,10,15,20$, and $25 \mathrm{~A} / \mathrm{m}$. The lines with $(\bullet)$ and the lines with $(\boldsymbol{A})$ are the results with and without thermal model in corporated, respectively.

resistance values are in good agreement. The measured negative conductance is $5.5 \times 10^{3} \mathrm{~A} /\left(\mathrm{V} \cdot \mathrm{cm}^{2}\right)$ at collector current density between 1.2 and $1.5 \times 10^{5} \mathrm{~A} / \mathrm{cm}^{2}$ [4]. The one shown in Fig. 5(a) is estimated (from curve of $\left.I_{b}=20 \mathrm{~A} / \mathrm{m}\right)$ to be $7.7 \times 10^{3} \mathrm{~A} /\left(\mathrm{V} \cdot \mathrm{cm}^{2}\right)$, which is in reasonable agreement with the measured value.

As explained previously, a smaller forward bias at the emitter-base junction is required to drive the same amount of base current when the junction temperature is higher. In the active region, $V_{b e}$ 's obtained from the thermal simulation are smaller than those calculated in the isothermal simulation. It indicates the base-emitter junction temperature is higher than the heat-sink temperature. In the saturation region $\left(V_{c e}<0.5 \mathrm{~V}\right), V_{b e}$ 's of the thermal simulation results are about the same as those of the isothermal case, indicating the base-emitter junction temperature is close to the heat-sink temperature.

The temperature distribution in the device operated at $V_{c e}$ of $3 \mathrm{~V}$ and $I_{b}$ of $20 \mathrm{~A} / \mathrm{m}$ is shown in Fig. 6(a). The largest temperature rise occurs in the collector region just beneath the emitter mesa. The corresponding distributions of the energy bands and the gradient of the energy flow $\left(\nabla \cdot \vec{J}_{E}\right)$, i.e., the power absorbed by the carriers per unit volume, in the region under the emitter mesa are shown

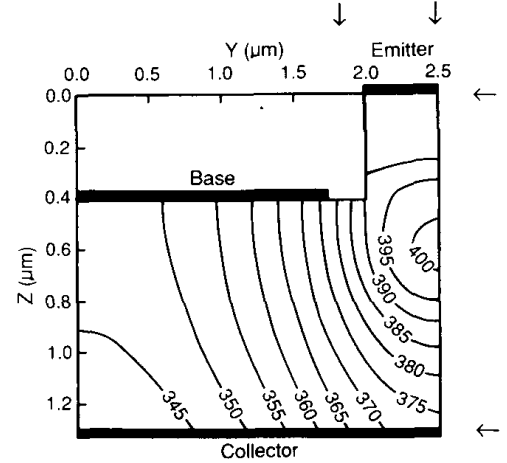

(a)

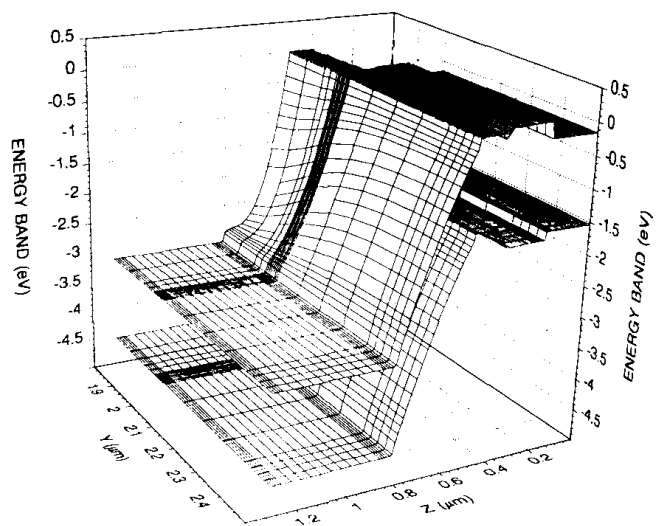

(b)

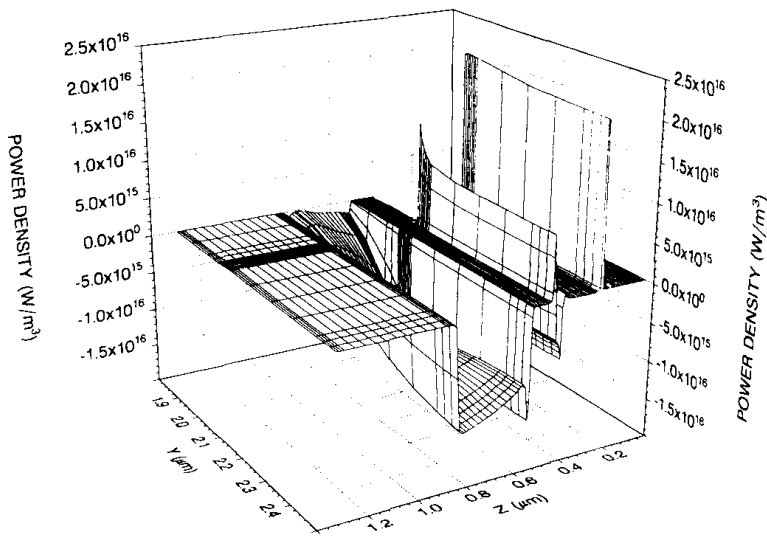

(c)

Fig. 6. (a) The contour plot of the calculated temperature distribution (temperature in Kelvin) of the HBT with the collector-emitter voltage of $3 \mathrm{~V}$ and the base current density of $20 \mathrm{~A} / \mathrm{m}$. The arrows point to the boundary of the region $(1.8 \mu \mathrm{m}<Y<2.5 \mu \mathrm{m} ; 0<Z<1.3 \mu \mathrm{m})$ in which the 3D figures are presented in (b) and (c). (b) The 3D plot of the conduction and valence band distributions. (c) The $3 \mathrm{D}$ plot of the absorption power per unit volume distribution.

in Fig. 6(b) and (c), respectively. A large power dissipation (shown in Fig. 6(c) as a negative quantity) occurs in the collector region. This is the Joule heating resulting from $\vec{E} \cdot \vec{J}$. It gives rise to a large temperature increase of about $100 \mathrm{~K}$ in this case. Fig. 7(a) shows the temper- 


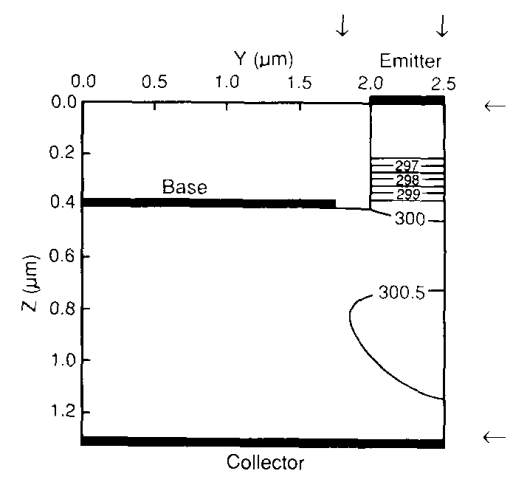

(a)

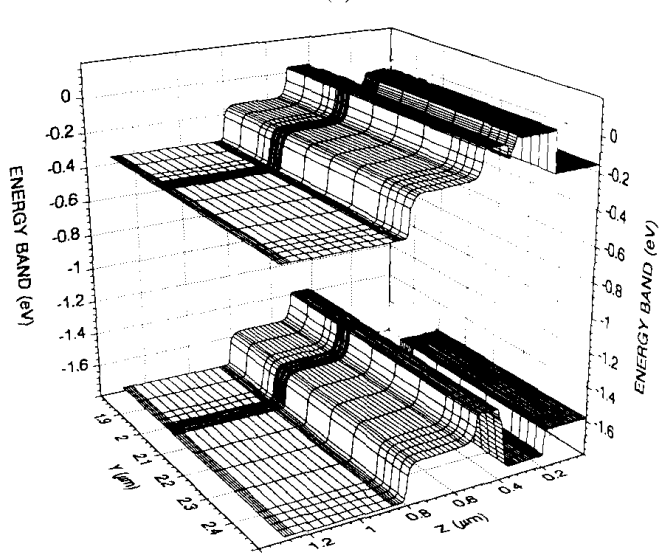

(b)

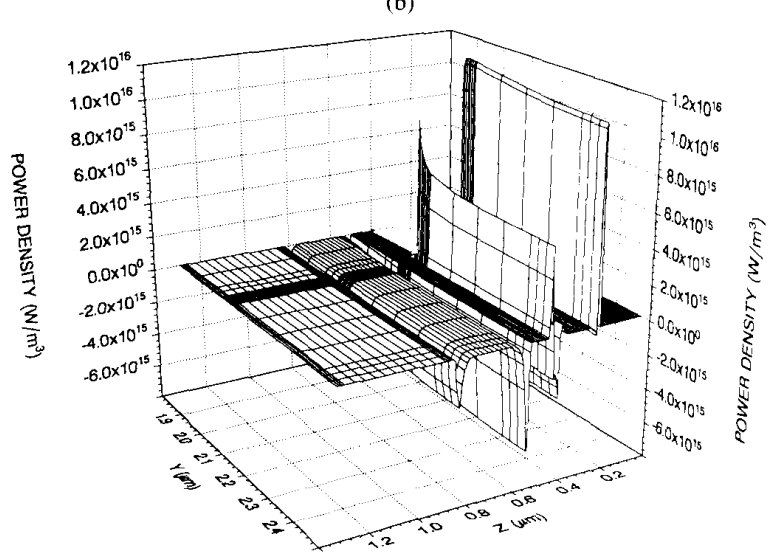

(c)

Fig. 7. (a) The contour plot of the calculated temperature distribution (temperature in Kelvin) of the HBT with the collector-emitter voltage of $0.2 \mathrm{~V}$ and the base current density of $20 \mathrm{~A} / \mathrm{m}$. The arrows point to the boundary of the region $(1.8 \mu \mathrm{m}<Y<2.5 \mu \mathrm{m} ; 0<Z<1.3 \mu \mathrm{m})$ in which the 3D figures are presented in (b) and (c). (b) The 3D plot of the conduction and valence band distributions. (c) The 3D plot of the absorption power per unit volume distribution.

ature contour for the device operated at $V_{c e}=0.2 \mathrm{~V}$ and $I_{b}=20 \mathrm{~A} / \mathrm{m}$. The corresponding spatial distributions of the energy bands and $\nabla \cdot \vec{J}_{E}$ in the cross section of the emitter mesa is shown in Fig. 7(b) and (c), respectively. Under this low applied voltage of $V_{c e}$, the base-collector junction is forward-biased, and the average electric field is small in the collector region. As a result, the power dissipation in the collector region is small, and the maximum temperature rise in the device is only $0.5 \mathrm{~K}$.

In Figs. 6(a) and 7(a), the temperature distributions in the emitter mesa region show that temperature decreases in the AlGaAs layer as the position approaches from the base layer toward the emitter contact. Fig. 7(a) shows that, while the base-emitter junction temperature is about the same as the heat-sink temperature, the emitter contact is abut $2.5 \mathrm{~K}$ less than that of the heat sink. This peculiar result originates from the large spike of the power absorption (shown as a positive quantity) at the heterojunction between the emitter cap and the wide-bandgap emitter as shown in Figs. 6(c) and 7(c). From the thermodynamic point of view [13], this Peltier-like effect is obtained when the energy flow equation (7) is used. The energy band discontinuities behaving as part of the thermoelectric power of the carriers affect heat flow with which the particle flow may occur under isothermal condition. From the particle point of view, the electrons gain energy through phonon absorption to overcome the energy barrier at the heterojunction. With the reduction of phonon population, the lattice temperature is consequently reduced. However, the basic assumption made here is the condition of the lattice subsystem to be in local thermal equilibrium with carriers. The depletion region and energy spike associated with the heterojunction may create nonequilibrium condition where the classical driftdiffusion model is no longer valid. A more detailed treatment incorporating hot-carrier effects is, therefore, needed in order to further understand the possible thermoelectric cooling effect in the heterostructure semiconductor devices.

\section{Summary}

We have studied the thermal effects on the HBT characteristics using two-dimensional numerical simulation. The NDR in the saturation region and the negative feedback of $V_{b e}$ for a constant base current are obtained. Thermal effects also contribute to the reduction of the current gain and cutoff frequency at high-power operation. The maximum temperature rise occurs in the collector region beneath the center of the emitter mesa. The results also show a possible thermoelectric cooling effect due to the heterojunction between the GaAs emitter cap and widebandgap $\mathrm{AlGaAs}$ emitter.

\section{APPENDIX}

The equivalent specific thermal resistance $R_{\text {th }}$ of the substrate depends not only on its thermal properties and geometry but also on the position of the heat source (active device). It is further complicated by the fact that the thermal conductivity is a function of temperature. It generally requires certain numerical techniques to calculate the value of $R_{\mathrm{th}}$. In the present study, the physical system is described in Fig. 1(b). The heat sink was assumed to 
be at the bottom of the substrate. The other surrounding surfaces are adiabatic, except for the heat source area. In this area, we assumed a uniform energy flow $Q$ which is calculated from the average of total device power output

$$
Q=\frac{\oint \vec{J}_{E} \cdot \hat{n} d a}{l_{x} l_{y}}
$$

where $\hat{n}$ is the outwardly normal unit vector, and $l_{x}$ and $l_{y}$ are the $x$ and $y$ dimensions of the bottom area of the device.

For the temperature-independent thermal conductivity case, the temperature distribution $\theta(x, y, z)$ is given in the following:

$$
\begin{aligned}
\theta(x, y, z)= & \Sigma^{\prime} a_{m n}\left(\sinh \gamma_{m n} z-\frac{\cosh \gamma_{m n} z}{\operatorname{coth} \gamma_{m n} d}\right) \cos \frac{2 \pi m x}{L_{x}} \\
& \cdot \cos \frac{2 \pi n y}{L_{y}}+\frac{Q l_{x} l_{y}}{\kappa_{0} L_{x} L_{y}}(z-d)+T_{0}
\end{aligned}
$$

where $\Sigma^{\prime}$ excludes the $m=n=0$ term, and

$$
\begin{aligned}
& \gamma_{m n}=2 \pi \sqrt{\left(m / L_{x}\right)^{2}+\left(n / L_{y}\right)^{2}} \\
& a_{m n}=\frac{4 Q}{m n \pi^{2} \kappa_{0} \gamma_{m n}} \sin \frac{m \pi l_{x}}{L_{x}} \sin \frac{n \pi l_{y}}{L_{y}}, \quad m, n \neq 0 \\
& a_{m 0}=\frac{2 Q l_{y}}{m \pi \kappa_{0} L_{y}} \sin \frac{m \pi l_{x}}{L_{x}} \\
& a_{0 n}=\frac{2 Q l_{x}}{n \pi \kappa_{0} L_{x}} \sin \frac{n \pi l_{y}}{L_{y}} .
\end{aligned}
$$

The $L_{x}, L_{y}$, and $d$ are the $x, y$, and $z$ dimensions of the substrate, respectively, and $\kappa_{0}$ is the thermal conductivity of the substrate at the heat-sink temperature. The Kirchhoff's transformation can be made to account for a temperature-dependent thermal conductivity [22]. Using $\kappa(T)$ $=\kappa_{0}(T / 300)^{-b}$, the corrected temperature $T$ is given by

$$
T=\left[\frac{1}{T_{0}^{b-1}}-\frac{(b-1)\left(\theta-T_{0}\right)}{T_{0}^{b}}\right]^{-1 /(b-1)}
$$

where $\theta$ is the uncorrected temperature. Using the reported data in [17], the best fitted value of $b$ is 1.22 . The equivalent specific thermal resistance is then calculated by

$$
R_{\mathrm{th}}=\frac{T_{\mathrm{av}}-T_{0}}{Q}
$$

where $T_{\mathrm{av}}$ is the average temperature at the heat source area, and is given by

$$
T_{\mathrm{av}}=\frac{\int T(x, y) d x d y}{l_{x} l_{y}} .
$$

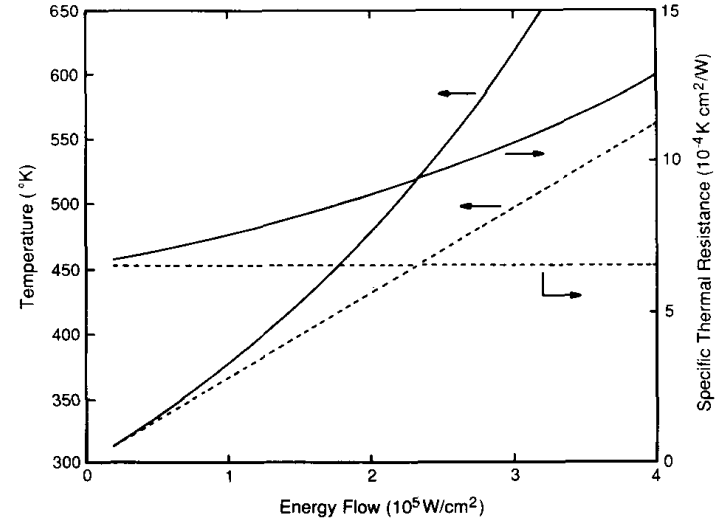

Fig. 8. The equivalent specific thermal resistance of the substrate and the average temperature at the interface between the device and the substrate as functions of the energy flow at the interface. Solid lines ( - ) and dashed lines (-- ) are values with and without the temperature-dependent thermal conductivity correction, respectively.

The calculated $R_{\mathrm{th}}$ and $T_{\mathrm{av}}$ as functions of $Q$ are shown in Fig. 8 for both temperature-dependent and temperatureindependent thermal conductivities in the substrate.

\section{ACKNOWLEDGMENT}

The authors wish to thank the reviewers for some helpful comments which lead to a better understanding of the subject presented here.

\section{REFERENCES}

[1] R. H. Winkler, "Thermal properties of high-power transistors," IEEE Trans. Electron Devices, vol. ED-14, pp. 260-264, 1967

[2] M. G. Adlerstein and M. P. Zaitlin, "Thermal resistance measurements for AlGaAs/GaAs heterojunction bipolar transistors," IEEE Trans. Electron Devices, vol. 38, pp. 1553-1554, 1991.

[3] J. R. Waldrop, K. C. Wang, and P. M. Asbeck, "Determination of junction temperature in AIGaAs/GaAs heterojunction bipolar transistors by electrical measurements," IEEE Trans. Electron Devices, vol. 39, pp. 1248-1250, 1992.

[4] D. S. Whitefield, C. J. Wei, and J. C. M. Hwang, "Temperaturedependent large signal model of heterojunction bipolar transistor,' to be published in Proc. 1992 IEEE GaAs IC Symp.

- "High power characterization and modeling of heterojunction bipolar transistors," to be published in Proc. IEEE Sarnoff Symp. 1992.

[5] M. E. Cheney, R. C. Fitch, G. D. Robinson, C. I. Huang, and R. A. Neidhard, "Electrical and thermal characteristics of heterojunction bipolar transistors fabricated on peeled film epitaxial layers," in Proc. Conf. on Advanced Concepts in High Speed Semiconductor Devices and Circuits, 1991, pp. 334-337.

[6] G-B Gao, M-Z Wang, X. Gui, and H. Morkoc,, "Thermal design studies of high-power heterojunction bipolar transistors," IEEE Trans. Electron Devices, vol. 36, pp. 854-863, 1989.

[7] G. Ghione and C. U. Naldi, "High-resolution self-consistent thermal modeling of multi-gate MESFETs," in IEDM Tech. Dig., 1989, pp. $147-150$.

[8] L. Liou, C. I. Huang, and J. Ebel, "Numerical studies of thermal effects on heterojunction bipolar transistor current-voltage characteristics using one-dimensional simulation," Solid-State Electron., vol. 35 , pp. 579-585, 1992.

[9] R. Stratton, IEEE Trans. Electron Devices, vol. ED-19, pp. 1288$1292,1972$.

[10] A. H. Marshak, "Transport equations for highly doped devices and heterostructures," Solid-State Electron., vol. 30, pp. 1089-1093, 1987. 
[11] G. H. Song, K. Hess, T. Kerkhoven, and U. Ravaioli, "'Two-dimensional simulator for semiconductor lasers," in IEDM Tech. Dig. 1989, pp. 143-146.

[12] D. C. Look, Electrical Characterization of GaAs Materials and Devices. New York: Wiley, 1989, pp. 83-85.

[13] G. K. Wachutka, "Rigorous thermodynamic treatment of heat generation and conduction in semiconductor device modeling," IEEE Trans. Computer-Aided Des., vol. 9, pp. 1141-1149, 1990.

[14] J. S. Blakemore, "Semiconducting and other major important properties of gallium arsenide," J. Appl. Phys., vol. 53, pp. R123-R181, 1982.

[15] R. J. Schuelke, "Numerical simulation of semiconductor heterostructure," Ph.D. dissertation, Purdue University, West Lafayette, IN, 1984.

[16] S. Adachi, "GaAs, AlAs, and $\mathrm{Al}_{x} \mathrm{Ga}_{1-x}$ As: Material parameters for use in research and device application," J. Appl. Phys., vol. 58, pp. R1-R29, 1985

[17] P. D. Maycock, "Thermal conductivity of silicon, germanium, III-V compounds and III-V alloys," Solid-State Electron., vol. 10, pp. 161$168,1967$.

[18] M. A. Afromowitz, "Thermal conductivity of $\mathrm{Ga}_{1-x} \mathrm{Al}_{x}$ As alloys," J. Appl. Phys., vol. 44, pp. 1292-1294, 1973.

[19] L. L. Liou, J. Ebel, and C. I. Huang, "DC transistor characteristics of mesa-etched-emitter heterojunction bipolar transistors using two dimensional simulation with current boundary condition," in Proc. NASECODE VII, 1991, pp. 241-242.

[20] G. B. Gao, Z. F. Fan, and H. Morkoc,, "Negative output differential resistance in AlGaAs/GaAs heterojunction bipolar transistors," Appl. Phys. Lett., vol. 61, pp. 198-200, 1992

[21] J. F. Palmier, J. Dangla, E. Caquot, and M. Campana, "Numerical simulation of electrical transport in III-V microstructure devices," in Proc. NASECODE I Conf., 1985, pp. 70-82.

[22] W. B. Joyce, "Thermal resistance of heat sinks with temperaturedependent conductivity," Solid-State Electron., vol. 18, pp. 321-322, 1975 .

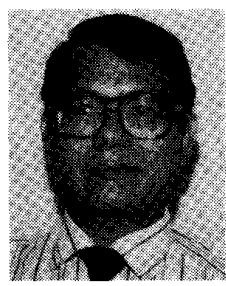

Lee L. Liou (S'82-M'87) was born in Taipei, Taiwan, in 1954. He received the B.S. degree in physics from National Tsing-Hua University, Hsin-Chu, Taiwan, in 1976, the M.S. degree in geophysics from National Taiwan University, Taipei, Taiwan, in 1978, and the Ph.D. degree in physics from University of Southern California Los Angeles, in 1985.

From 1985 to 1986 , he was with Hewlett-Packard Fort Collins Integrated Circuit Division, Ft Collins, CO, where he was responsible for ad- vanced CMOS metrology development. From 1987 to 1989 , he was with Universal Energy System Inc., Dayton, OH, where he was involved in the development of the advanced heterojunction devices for high-speed communiction applications. Since 1990, he has been with Solid-State Electronics Directorate, Wright Laboratory, Wright-Patterson Air Force Base, OH. $\mathrm{He}$ is presently working on the modeling of power heterojunction bipolar transistors.

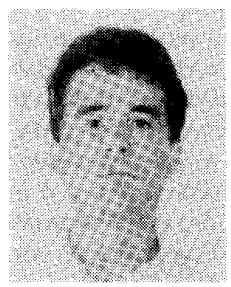

John L. Ebel received the B.S. and M.Eng. degrees in electrical engineering from Cornell University, Ithaca, NY, in 1984 and 1985 , respectively.

He was commissioned a 2 nd Lieutenant in the Air Force in 1984, and was stationed at the Avionics Laboratory at Wright-Patterson Air Force Base with the Design Group of the Microelectronics Branch through February 1989. During that time, he worked on development CAD tools, languages (VHDL), and environments as part of the Very High Speed Integrated Circuits Program (VHSIC). He is currently working towards the Ph.D. degree at Carnegie Mellon University, Pittsburgh, PA.

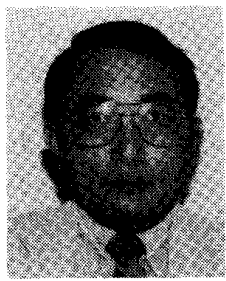

Chern I. Huang (M'74) was born in Taiwan in 1940. He received the B.S.E.E. degree from $\mathrm{Na}$ tional Cheng-Kung University, Taiwan, in 1962, the M.S.E.E. degree from Iowa State University, Ames, in 1967, and the Ph.D. degree in electrica engineering from University of Florida, Gaines ville, in 1973 .

He had worked for Collins Radio Company and Bendix Avionics Division between 1966 and 1969. Since 1973, he has been with the Materials, Avionics, and Solid State Electronics Directorates, Air Force Wright Laboratory, Wright-Patterson Air Force Base, $\mathrm{OH}$, on various assignments: in-house research, project management, and administrative duties. His research and development interests have been in compound semiconductor materials, device physics and modeling, and circuits for microwave and digital applications. 